\title{
A Centralized Displacement Operation with Application to Artifact Reduction in Ultrasonic Elastography
}

\author{
Dangguo Shao ${ }^{1 *}$, Yong Chen ${ }^{\mathrm{b}}$, Sanli $\mathrm{Yi}^{1}$,Lei Ma ${ }^{1}$,Jianfeng $\mathrm{He}^{1}$ and Dong C. Liu ${ }^{2}$ \\ ${ }^{1}$ Faculty of Information Engineering and Automation, KunMing University of Science \\ and Technology, KunMing, China \\ ${ }^{2}$ School of Computer Science, Sichuan University, Chengdu, China \\ *Corresponding author: huntersdg@126.com
}

\begin{abstract}
Because stiff tissue deforms less than soft tissue under the same external compression, elastography can provide relative stiffness information of biological tissue. However, elastography suffers from artifact noise which may come from two dominant sources: decorrelation error and amplitude modulation error. In order to reduce artifacts and improve the quality of ultrasonic elastography, this paper proposes a centralized displacement operation based on an adaptive anisotropic diffusion filtering. We have applied the median and the mean of displacement axial gradient to differentiate edges from artifacts and categorize the whole displacement image into two different patterns; followed by the adjustable anisotropic diffusion filtering. The proposed algorithm can reduce artifact noise and, at the same time, maintain the tissue structure. Phantom testing shows that the proposed method can improve the quality of ultrasonic elastography in terms of tissue SNRe and CNRe values.
\end{abstract}

Keywords: Ultrasonic Elastography, Artifact Reduction, Displacement, SNRe, CNRe

\section{Introduction}

Elastography or strain imaging, the imaging modality of elastic properties of biological tissue using ultrasound, was first presented by Ophir et al. in 1991[1]. Because stiff tissue deforms less than soft tissue under the same external compression, elastography can provide relative stiffness information of biological tissue. This imaging modality has been shown to be useful in different clinical applications [2-5].

However, elastography suffers from artifact noise which may come from two dominant sources: de-correlation error and amplitude modulation (AM) error. De-correlation error is caused by compression, deformation, slide-slippage, and other movement sources, which makes the motion tracking not follow the conventional time delay estimation model. AM error is caused by the random fluctuation of signal amplitude, which makes the location of the displacement estimate not at the center of the analysis window. Some research groups have tried to improve elastogram performance by using various approaches, such as temporal stretching [6-8], adaptive stretching [9], wavelet de-noising [10], amplitude modulation correction [11] and compounding approach [12-17].

A filtering technique, to be called a centralized displacement operation based on an adaptive anisotropic diffusion filtering, is presented to reduce artifact noise in this study. The median and mean of displacement gradient differentiates edge from homogenous region and categorize the whole image into two different feature patterns; followed by the adjustable anisotropic diffusion filtering. 
The organization of the paper is as follows. Section 2 describes the theoretical basis and our method. Methods are demonstrated in Section 3 using phantom data and are evaluated using quantitative quality metrics. Conclusions and potential future directions are presented in Section 4.

\section{Method}

\subsection{Centralized Displacement}

A strain image can be produced by displaying spatial derivatives from the displacement (time shift) estimates. But in fact, the estimate of the time shift between two signals obtained before and after target compression is affected by the signal amplitude. If the strain estimates are obtained directly from the time shift measurement, this effect results in an undesired random modulation of the strain estimates by the random signal envelope amplitude. Hence, a displacement estimate does not often closely track displacement at the window center. The actual location is skewed towards higher amplitude portions of the windowed signal [6]. The distribution of signal envelope amplitudes follows some statistical distribution (e.g., Rayleigh if there is a large number of small randomly distributed scatterers [18]), and the added variability due to the strain modulation effect is related to that envelope amplitude distribution. In general, the signal envelope amplitude is independent of the elasticity of the medium; the elastographic noise introduced by the undesirable leakage of signal envelope amplitude information into the strain estimations is considered an artifact [8]. This is why location estimation error is one of sources of noise [11, 19].

For the sake of clarity, we analyze the simplest estimate of 1-D strain, e, where 1-D displacement estimates are differenced and divided by the spacing between them:

$$
e=\frac{\Delta d}{\Delta l}=\frac{d_{2}-d_{1}}{l_{2}-l_{1}}
$$

where $d_{1}$ and $d_{2}$ are displacement estimates from windows 1 and 2 respectively, and $l_{1}$ and $l_{2}$ record the estimation locations, which are not in general the same as the centers of the windows. It is sometimes assumed that (1) contains only two random variables, $d_{1}$ and $d_{2}$. Lindop [11] examines the other variables, 12 and 11. There are two sources of estimation noise in strain imaging, de-correlation error and the location estimation error. AM is often the primary source of artifact noise in ultrasonic strain images where it is not corrected or suppressed [11].

If the strain image is calculated from the actual displacement and its actual location, most of location estimation errors can be reduced. However, the location estimation error will be introduced when the strain image is calculated from the actual displacement and its ideal location which is the center of window. In order to reduce the location estimation error in strain imaging, the actual displacement will need a centralized displacement operation. The latter will make the former to be close to an ideal displacement at the center of window.

Various types of filter can be applied to centralize displacement operation. In this study, we choose an anisotropic diffusion filter which is smoothing image at the same time preserving edges.

\subsection{Centralized Displacement Operation}

On continuous domain, an anisotropic diffusion method is as follows [20,21], 


$$
\left\{\begin{array}{c}
\frac{\partial I}{\partial t}=\operatorname{div}[c(|\nabla I|) \cdot \nabla I] \\
I(t=0)=I_{0}
\end{array}\right.
$$

where $\nabla$ is the gradient operator, div is the divergence operator, $\|$ denotes the magnitude, c(.) is the diffusivity function, and $\mathrm{I} 0$ is the initial image. According to a need, a suitable diffusivity function will be required to be chosen. In displacement image, the diffusivity function is as follows.

$$
c(x)=1 /\left(1+\left(\frac{x}{k}\right)^{2}\right)
$$

The discretization formation is as follows.

$$
I_{s}^{t+1}=I_{s}^{t}+\lambda \sum_{p \in \eta_{s}} c\left(\nabla I_{s, p}^{t}\right) \nabla I_{s, p}^{t}
$$

where $I_{s}^{t}$ denotes the gray value of a pixel which position is $s$ in a 2-D image. When $\nabla I$ is large, that is the more noise region to be smoothed. When $\nabla I$ is small, that is the less noise region to reduce the smoothness to keep details. $\lambda$ is the TSS which value is from 0 to 0.25 . Because (4) is an explicit discretization scheme, in order to keep the stability of above equation, the value of TSS must be very small.

There are two features for displacement image. Firstly, the values of displacement image are order from up to down; secondly, the gradient of soft tissue region is larger than that of hard cyst region. According to the formation (3), the value of $k$ can be as threshold to adjust smoothing in anisotropic diffusion filtering. In a traditional anisotropic diffusion filter, the value of $k$ is a fixed value for all filtering steps, but it is relatively difficult to select the value of $k$ for a displacement image. Therefore, the fixed value of $k$ is not appropriate for every filtering step. The median and the mean of axial gradients in displacement image are two values between the larger gradient and the smaller gradient. The median and the mean of displacement gradient can roughly differentiate edge region from homogenous region. In this paper, the value of $k$ in every filtering step is adapted by the following formation.

$$
k^{t+1}=\frac{\text { median }\left(\nabla I^{t}\right)+\text { mean }\left(\nabla I^{t}\right)}{m}
$$

where $m$ denotes a constant from 1.0 to 2.0. The classified result is used in an adaptive anisotropic diffusion filter so that adaptive smoothing for artifact reduction is achieved. In addition, the user can adjust the number of iteration to fine-tune smoothing in different applications.

\subsection{The Algorithm}

The algorithm of centralized displacement operation is as follows:

1) Input displacement image of a size of $\mathrm{M} \times \mathrm{N}$, l;

2) Initialize parameters, for example, $\lambda$ (such as $1 / 7$ ), $m$ (such as 1.2) and the number of iterations (such as 30);

3) Calculate the axial gradient matrix of displacement image, $\nabla \mathbf{l}$; 
4) According to the formula (5), calculate the threshold, $k$;

5) According to the formulas (3) and (4), calculate $I_{i j}{ }^{t+1}, i \in M, j \in N$, where $t$ is the number of iterations;

6) Meet the maximum number of iterations then stop; otherwise, continue;

7) Output the centralized displacement image, $I^{t+1}$.

\section{Results and Discussion}

\subsection{Criteria for Quantifying Algorithm Performance}

Two main image quality metrics in ultrasonic elastography, SNRe [16, 17] and CNRe [16], are applied to both simulation and phantom images. The elastographic signal-to-noise ratio (SNRe) quantifies the level of noise as the ratio of mean to standard deviation of strain values. And the elastographic contrast-to-noise ratio (CNRe) quantifies the level of contrast between a region of interest and the background. They are calculated as:

$$
\begin{gathered}
S N \operatorname{Re}=\frac{\mu}{\sigma} \\
C N \operatorname{Re}=\frac{2 \cdot\left(\mu_{t}-\mu_{b}\right)^{2}}{\sigma_{t}^{2}+\sigma_{b}^{2}}
\end{gathered}
$$

where $\mu_{t}$ is the mean of a region of interest, and $\mu_{b}$ and $\sigma_{b}$ are the mean and variance of similar sized regions in the image background. upSNRe and upCNRe, which are the growth rates of two image quality metrics, are defined as follows.

$$
\begin{aligned}
& \text { upSN } \operatorname{Re}=100 \cdot\left(\frac{S N \mathrm{Re}_{\text {result }}}{S N \operatorname{Re} e_{\text {original }}}-1\right) \\
& \text { upCN } \operatorname{Re}=100 \cdot\left(\frac{C N \operatorname{Re}_{\text {result }}}{C N \operatorname{Re}_{\text {original }}}-1\right)
\end{aligned}
$$

\subsection{Phantom Image}

We have verified our proposed algorithms using a phantom ultrasonic elastography obtained from Saset Healthcare iMago C21, a commercial digital ultrasound scanner. In phantom experiment, a $5.0 \mathrm{MHz}$ center frequency linear array probe is used for data acquisition and freehand probe compression is applied. Sampling frequency is $40 \mathrm{MHz}$.Decimated IQ data of 512 samples per line is used to calculate the elastogram. We form 188 beam lines for this scan. The Model 049 Elasticity QA phantom, CRIS Virginia, USA, is used in our experiment to test the algorithm. The strain images are obtained from Phase Zero method [22]. The axial strain is calculated by using the 1-D low-pass digital differentiator [23]:

$$
y(n)=\sum_{k=1}^{M} \frac{x(n+k)-x(n-k)}{2 k \cdot M}
$$


The results are shown by Figure 1, which is a phantom ultrasonic elastography. Figure 1a is an original displacement image from phantom. Figure $1 \mathrm{~b}$ is produced by displaying spatial derivatives from Figure 1a. Figure 1c is produced by anisotropic diffusion filtering from Figure 1b. Figure 1d is produced by anisotropic diffusion filtering from Figure 1a. Figure 1e is produced by displaying spatial derivatives from Figure 1d. Table 1 shows SNRe and CNRe values derived from a white square $(70 \times 70$ pixels $)$ and a black square $(70 \times 70$ pixels $)$ respectively. Figure 1b, c and e display examples of these regions using white and black squares to indicate the background and region of interest respectively.

\subsection{Discussion}

The strain image of centralized displacement (Figure 1e) are shown better contrast than the filtered strain image (Figure 1c) when remaining useful clinical information. Quantitatively, from Table 1, both CNR and SNR from the strain image of centralized displacement (Figure 1e) are higher than that from the filtered strain image. On the other hand, the centralized displacement operation in ultrasonic elastography ( 0.771424 seconds) is faster to make for real time than the filtering strain image (18.398092 seconds), because the size of displacement image $(161 \times 188$ pixels $)$ is smaller than the size of strain image $(512 \times 460$ pixels), and the number of iteration in the centralized displacement operation (30 steps) is fewer the number of iteration in the filtering strain image (100 steps). From the artifact reduced strain images, the method of this paper is efficient to reduce the artifact noise and, at the same time, maintain the tissue structure.

Table 1. SNRe and CNRe of a Ultrasonic Elastography

\begin{tabular}{|c|c|c|}
\hline strain image & smoothed strain (Fig. 1c) & $\begin{array}{l}\text { smoothed displacement(Fig. } \\
1 \mathrm{e})\end{array}$ \\
\hline $\mathrm{k}$ & 5.0 & adaptive \\
\hline $\mathrm{t}$ & 100 & 30 \\
\hline time(s) & 18. 398092 & 0.771424 \\
\hline $\mathrm{SNRe}_{\text {original }}$ & 7.912174 & 7. 912174 \\
\hline $\mathrm{SNRe}_{\text {result }}$ & 11.733238 & 12. 172589 \\
\hline upSNRe (\%) & 48. 293472 & 53. 846329 \\
\hline $\mathrm{CNRe}_{\text {original }}$ & 17.748552 & 17.748552 \\
\hline $\mathrm{CNRe}_{\text {result }}$ & 46.634167 & 51.355387 \\
\hline upCNRe (\%) & 162.749141 & 189. 349735 \\
\hline
\end{tabular}


International Journal of Signal Processing, Image Processing and Pattern Recognition Vol.7, No.2 (2014)

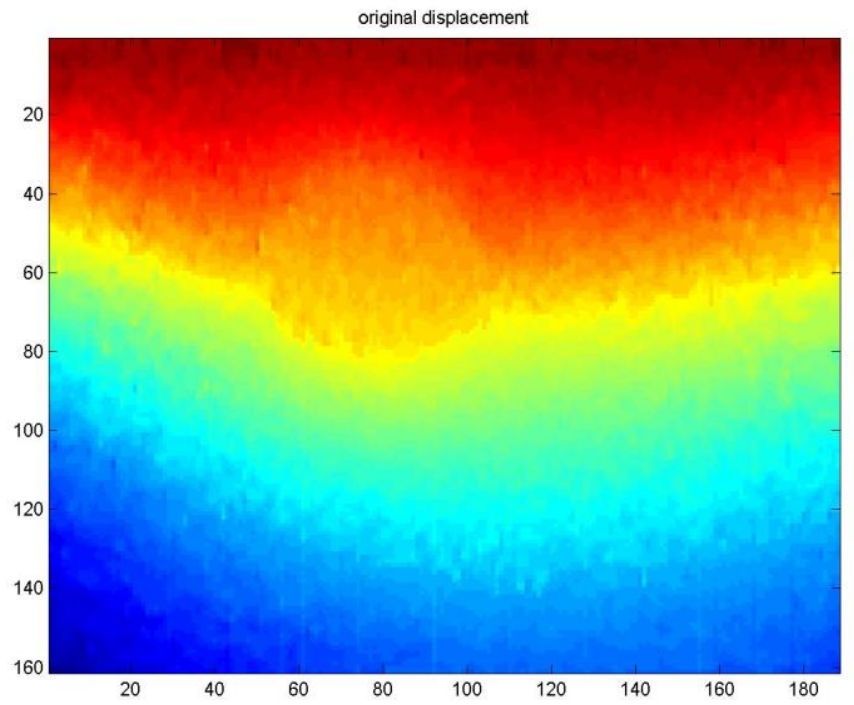

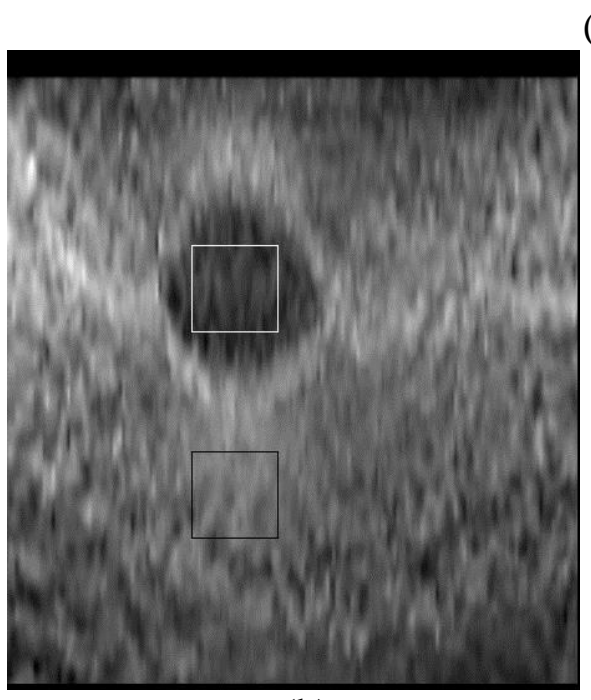

(b)

(a)

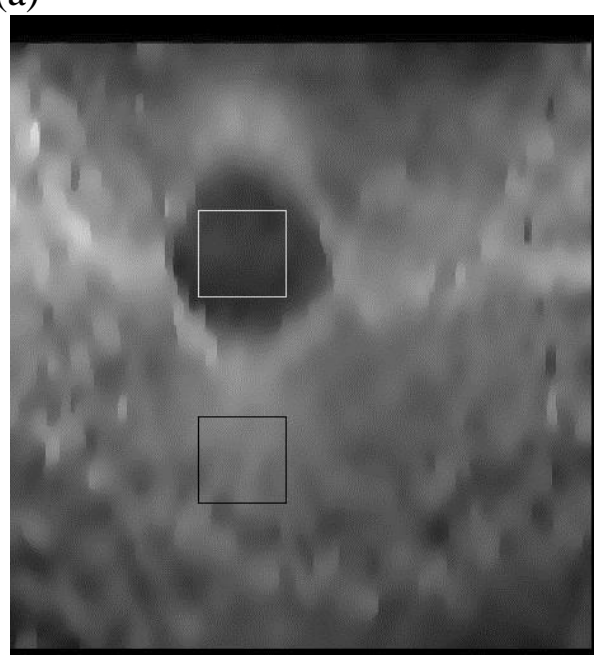

(c) 


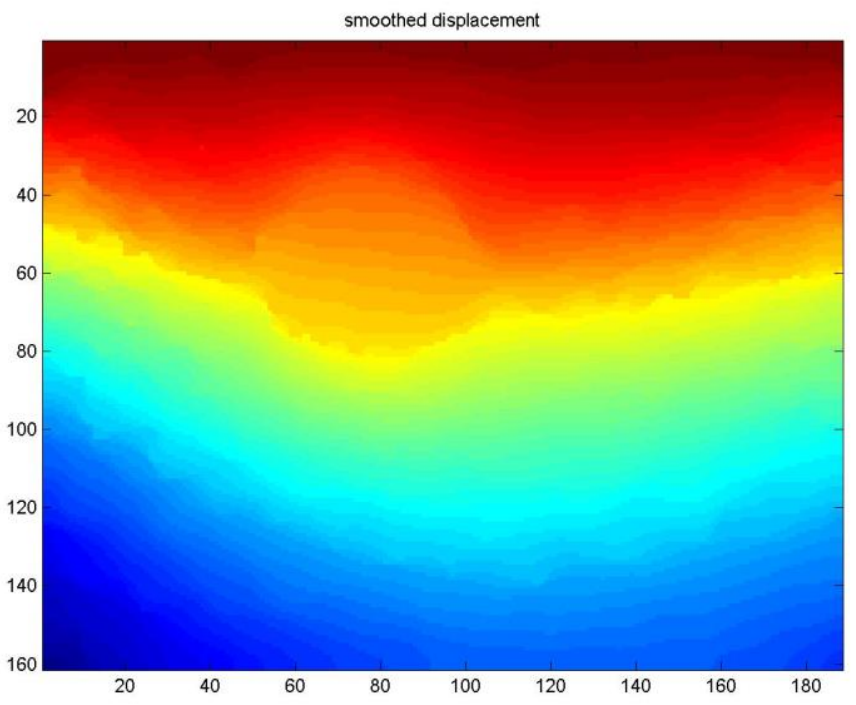

(d)

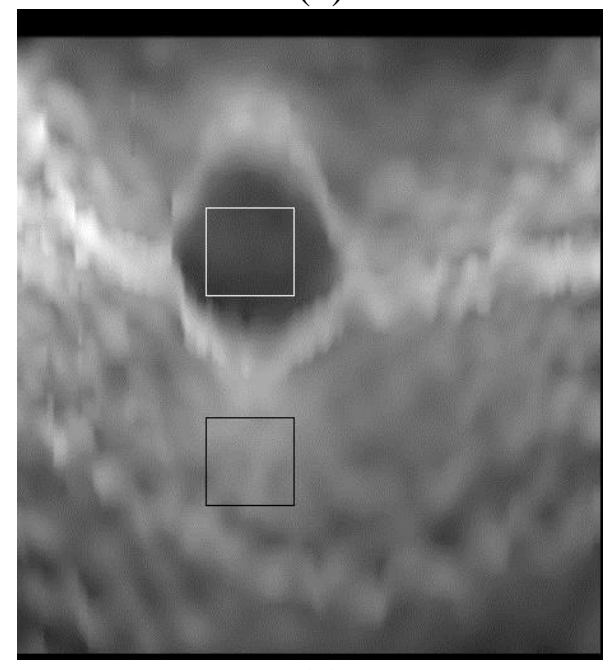

(e)

Figure 1. The Elastogram Obtained from Model 049 Elasticity QA Phantom.

Window Length is 10 samples with a $\mathbf{7 5 \%}$ Overlap. M is 7 in Low-Pass Digital

Differentiator. (a) Original Displacement Image. (b) Original Strain Image. (c) The Filtered Strain Image by Anisotropic Diffusion Filter. (d) The Filtered

Displacement image by Anisotropic Diffusion Filter. (e) The Strain Image from (d)

\section{Conclusion}

Ultrasonic elastography is an imaging technique providing relative stiffness information of biological tissues. In general, elastography will suffer from artifacts noise, which degrades lesion detectability and increases the likelihood of misdiagnosis. The way to suppress artifacts effectively and preserve the structure is vital for diagnosis and also for image post-processing. In order to reduce artifacts and improve the quality of ultrasonic elastography, this paper 
proposes a centralized displacement operation base on an adaptive anisotropic diffusion filtering. We have applied the median and the mean of displacement axial gradient to differentiate edges from artifacts and categorize the whole displacement image into two different patterns; followed by the adjustable anisotropic diffusion filtering. Testing in phantom imaging demonstrates that our method can reduce artifacts noise and preserve the structure at the same time. Currently, we are looking for the way to speed up the processing both in DSP and GPU platforms. Some in vivo elastographys will be tested for fine-tune algorithms and parameters optimization. Further investigation into breast and liver elastographys is necessary.

\section{Acknowledgements}

The authors extend thanks to Jie Ren, Congyao Zhang and Shaoguo Cui for many valuable suggestions during the algorithm development and testing in phantom elastographys.

\section{References}

[1] J. Ophir, I. Céspedes, H. Ponnekanti, Y. Yazdi and X. Li X, "Elastography: A quantitative method for imaging the elasticity of biological tissues", Ultrason Imaging, vol. 13, (1991), pp. 111-134.

[2] E. E. Konofagou, J. D’hooge and J. Ophir, "Myocardial elastography—A feasibility study", Ultrasound Med. Biol, vol. 28, no. 4, (2002), pp. 113-132.

[3] T. Varghese, J. Zagzebski and J. Jee, "Elastography imaging of thermal lesion in the liver in vivo following radio frequency ablation: Preliminary results", Ultrasound Med. Biol., vol. 28, (2002), pp. 1467-1473.

[4] A. Thitaikumar, L. M. Mobbs, C. M. Kraemer-Chant, B. S. Garra and J. Ophir, "Breast Tumor Classification using axial shear strain elastography: a feasibility study", Phys. Med. Biol., vol. 53, (2008), pp. 4809-4823.

[5] K. Thittai Arun, G. Belfor and O. Jonathan, "Importance of axial compression verification to correct interpretation of axial-shear strain elastograms in breast lesions", Ultrasonic Imaging, vol. 32, (2010), pp. 190-198.

[6] I. Cespedes and J. Ophir, "Reduction of image noise in elastography", Ultrason. Imag., vol. 15, (1993), pp. 89-102.

[7] T. Varghese, J. Ophir and I. Cespedes, "Noise reduction in elastograms using temporal stretching with multicompression averaging", Ultrasound Med. Biol., vol. 22, (1996), pp. 1043-1052.

[8] S. K. Alam and J. Ophir, "Reduction of signal decorrelation from mechanical compression of tissues by temporal stretching: Applications to elastography"' Ultrasound Med. Biol., vol. 23, (1997), pp. 95-105.

[9] S. K. Alam, J. Ophir and E. E. Konofagou, "An adaptive strain estimator for elastography", IEEE Trans Ultrason Ferroelec Freq Control, vol. 45, no. 2, (1998), pp. 461-472.

[10] U. Techavipoo and T. Varghese, "Wavelet de-noising of displacement estimates in elastography", Ultrasound Med. Biol., vol. 30, no. 4, (2004), pp. 477-491.

[11] J. E. Lindop, G. M. Treece, A. H. Gee and R. W. Prager, "Estimation of Displacement Location for Enhanced Strain Imaging", IEEE Transactions on Ultrasonics, Ferroelectrics, and Frequency Control, vol. 54, no. 9, (2007), pp. 1751-1771.

[12] U. Techavipoo, Q. Chen, T. Varghese, J. A. Zagzebski and E. L. Madsen, Noise reduction using spatialangular compounding for elastography", IEEE Trans Ultrason Ferroelec Freq Control, vol. 51, no. 5, (2004), pp. 510-520.

[13] K. Zhang, P. Liu and D. C. Liu, "Strain Image with Spatial Angular Compounding", The 4th International Conference on Bioinformatics and Biomedical Enginerring ICBBE, (2010).

[14] Y. Cheng, S. Cui, P. Liu and D. C. Liu, "Frequency Compounding for Ultrasound Freehand Elastography", The 4th International Conference on Bioinformatics and Biomedical Engineering, ICBBE (2010).

[15] W. Liu, Y. Cheng, P. Liu and D. C. Liu, "Filter Based Spatial Compounding for Strain Imaging", Proceedings of 2010 IEEE 17th International Conference on Image Processing, Hong Kong, (2010).

[16] S. Cui and D. C. Liu, "Noise Reduction for Ultrasonic Elastography Using Transmit-Side Frequency Compounding: A Preliminary Study”, IEEE Trans Ultrason Ferroelec Freq Control, vol. 58, no. 3, (2011), pp. 509-516.

[17] P. Liu and D. Liu, "Filter-Based Compounded Delay Estimation With Application to Strain Imaging", IEEE Trans Ultrason Ferroelec Freq Control, vol. 58, no. 10, (2011), pp. 2078-2095.

[18] V. Dutt and J. F. Greenleaf, “Adaptive Strain noise Reduction Filter for Log-Compressed B-Scan Images”, IEEE Trans. Medical Imaging, vol. 15, (1996), pp. 802-813. 
[19] J. E. Lindop, G. M. Treece, A. H. Gee and R. W. Prager, "Phase-based ultrasonic deformation estimation", IEEE Transactions on Ultrasonics, Ferroelectrics and Frequency Control, vol. 55, no. 1, (2008), pp. 94-111.

[20] F. Torkamani-Azar and K. E. Tait, "Image Recovery Using the Anisotropic Diffusion Equation”, IEEE Trans. on Image Processing, vol. 5, (1996), pp. 1573-1578.

[21] H. G. Luo, L. M. Zhu and H. Ding, "Coupled anisotropic diffusion for image selective smoothing", Signal Processing, vol. 86, no. 7, (2006), pp. 1728-1736.

[22] A. Pesavento, C. Perrey, M. Krueger and H. Ermert, "A time-efficient and accurate strain estimation concept for ultrasonic elastography using iterative phase zero estimation", IEEE Trans Ultrason Ferroelectr Freq Control, vol. 46, no. 5, (1999), pp. 1057-1067.

[23] J. Luo, J. Bai, P. He and K. Ying, "Axial strain calculation using a low-pass digital differentiator in ultrasound elastography”, IEEE Trans. Ultrason. Ferr. Freq. Control, vol. 51, no. 9, (2004), pp. 1119-1127. 
International Journal of Signal Processing, Image Processing and Pattern Recognition Vol.7, No.2 (2014) 